England between 2010 and 2013. Participants comprised 567 children presenting with unintentional poisoning occurring at home, and 2320 control participants matched on age, sex, date of event and study centre. Parents/caregivers provided data on safety practices, safety equipment use, home hazards and potential confounders, by means of self-completion questionnaires. Data were analysed using conditional logistic regression.

Results Compared with controls, parents of poisoned children were significantly more likely not to store medicines out of reach (adjusted odds ratio (AOR) 1.59; 95\% CI: 1.21, 2.09; population attributable fraction (PAF) 15\%), not to store medicines safely (locked or out of reach (AOR 1.83; 95\% CI: 1.38, 2.42; PAF $16 \%$ ) and not to have put all medicines (AOR 2.11; 95\% CI: 1.54, 2.90; PAF 20\%) or household products (AOR 1.79, 95\% CI: $1.29,2.48$; PAF $11 \%$ ) away immediately after use.

Conclusions Not storing medicines out of reach or locked away and not putting medicines and household products away immediately after use increased the odds of secondary care attended poisonings in 0-4 year olds. If associations are causal, implementing each of these poison prevention practices could prevent between $11 \%$ and $20 \%$ of poisonings.

\section{MATERNAL POSTPARTUM DEPRESSION IS ASSOCIATED WITH INCREASED RISK OF EARLY CHILDHOOD INJURY}

${ }^{1,2}$ Dustin W Currie, ${ }^{1}$ Claire Colangelo, ${ }^{1}$ Mallory Quigley, ${ }^{1}$ Alexa Cares, ${ }^{1}$ Molly O'Fallon,
${ }^{1}$ William Thorland. ${ }^{1}$ The Nurse-Family Partnership National Service Office, Denver, CO;
${ }^{2}$ Program in Injury Prevention, Education and Research (PIPER), Department of
Epidemiology, Colorado School of Public Health, Aurora, CO 10.1136/injuryprev-2016-042156.401

Background Previous research indicates an association between maternal postpartum depression (PPD) and child maltreatment, but research on this association in child injury outside of the maltreatment context is limited. The Nurse-Family Partnership (NFP) is a nurse home visiting program in which nurses visit first-time, low-income moms from pregnancy until the child's second birthday, promoting healthy parenting practices. As early childhood injury or ingestion emergency room (ER) visits are primary NFP outcomes, there is an interest in determining risk factors for these outcomes within the client population.

Methods We analysed a cohort of NFP clients beginning the program between 7/1/2010 and 6/30/2012 with completed 12month infant health care and PPD forms $(n=6271)$. Postpartum depression was measured using the Edinburgh Postnatal Depression Scale (EPDS) administered 1 to 8 weeks after birth, with a cutoff score of $\geq 10$ indicating possible PPD. The outcome was defined as self-reported infant ER visit due to injury or ingestion on the 6- or 12-month infant health care form. Logistic regression was used to calculate adjusted odds ratios of the relationship between PPD and injury.

Results A total of 985 clients (15.8\%) exceeded the possible PPD cutoff on the EPDS. Injury- or ingestion-related ER visits in the child's first year of life were reported by 385 clients (6.2\%). Postpartum depression scores above the cutoff were significantly associated with ER visits after adjusting for child gender and maternal race, age, education and marital status (adjusted OR: 1.41 [1.07, 1.84], $\mathrm{p}=0.01)$.

Conclusions We found a significant association between PPD and subsequent risk of childhood injury/ingestion ER visit within a cohort of NFP clients after adjusting for related confounders. Understanding how postpartum depression affects early childhood outcomes may provide opportunities for targeted injury prevention interventions within the home visitation program context.

\section{CHILD INJURIES IN CROATIA - SIGNIFICANT PUBLIC HEALTH ISSUE}

Ivana Brkic Bilos, Maja Silobrcic Radic, Verica Kralj, Tanja Coric. Croatian Institute of Public Health, Croatia

\subsection{6/injuryprev-2016-042156.402}

Background Injuries are the leading cause of death among children in Croatia. In recent decades, the work on monitoring and prevention of injuries has intensified, which led to a reduction of child mortality caused by injuries.

Methods The data used in the research was collected from the routine mortality and morbidity statistics, the database of the World Health Organisation: European Detailed Mortality Database (DMDB), as well as from the results of the international project TACTICS (Tools to Address Childhood Trauma, Injury and Children's Safety).

Results In Croatia, in the last ten-year period from 2005 to 2014 has been noted a trend of reducing the mortality rates from total number of injuries (V01-Y98) among children aged 0-19. The highest age-specific death rate from injuries in children in the mentioned period was 14.6/100.000 (2005), and the lowest 7.1/100.000 (2014), which represents almost double reduction in mortality. The reason for this is primarily the trend of reducing the mortality rate due to traffic accidents (V01-V99) in children (8.6/100.000 (2005); 2.6/100.000 (2014)). The leading external causes of death from injury in the observed period were: traffic accidents, suicide, drowning, followed by poisoning and suffocation. According to the European DMDB database, Croatia has been positioned around the middle of the European scale with the standardised mortality rate from injury (0-19 years) 8.8/100 000 (2012). According to the research carried out in the framework of the international project TACTICS, it was noted that Croatia has a medium satisfactory child safety level in the area of unintentional child injury prevention. In the violence prevention area Croatia belongs to the European countries with progressive politics.

Conclusions Although in the last few decades Croatia perceives a trend of reducing mortality from child injuries, further efforts in the area of monitoring, treatment and prevention of child injuries are needed.

\section{CHILD PEDESTRIAN COLLISIONS, WALKING TO SCHOOL AND THE BUILT ENVRIONMENT: A CASE CONTROL STUDY}

1,2Linda Rothman, ${ }^{2}$ Alison Macpherson, 2,3Ron Buliung, 1,2 Sarah Richmond, ${ }^{2}$ Colin Macarthur, ${ }^{1}$ Andrew Howard. ${ }^{1}$ York University, Toronto, Canada; ${ }^{2}$ Hospital for Sick Children Toronto, Canada; ${ }^{3}$ University of Toronto Mississauga, Mississauga, Canada

\subsection{6/injuryprev-2016-042156.403}

Background Walking to school is a way to increase daily physical activity; however the risk of injury must also be considered so that walking does not lead to an increase in pedestrian injuries. Risk factors associated with the environment around schools with high child pedestrian motor vehicle collision (PMVC) rates were examined.

Methods Child PMVCs from 2000-2013, ages 4-12 years, were mapped within elementary school attendance boundaries in 